\title{
Commentary: Factors affecting patient choice for continued observation versus intervention for pelvic organ prolapse
}

\author{
Alexandra Dubinskaya ${ }^{1}$ (D) Kaitlin Renkosiak $^{2}$ \\ Received: 8 August 2020 / Accepted: 24 August 2020 / Published online: 5 September 2020 \\ (C) The International Urogynecological Association 2020
}

As the population ages, the prevalence of women with pelvic organ prolapse (POP) increases. Little is known about the natural progression of prolapse but it is believed to progressively worsen over time. Subjective symptoms typically guide management. However, factors that influence patients to choose intervention versus observation are not well understood.

In this retrospective study performed at the Medical University of South Carolina, 73 women who were diagnosed with POP elected to continue with observation. Patients were observed between 6 and 36 months. After the last recorded clinic visit, subjects were further divided into two groups, those who chose an intervention (pessary or surgery) and those who continued observation. These two groups were compared, and factors that influenced the choice for intervention, as well as anatomical and functional changes, were assessed. Notably, only $11 \%(8 / 73)$ of women in the observation group had worsening symptoms compared to $53 \%(20 / 38)$ in the intervention group. No difference in worsening of POP-Q stage was found. However, an increase in the leading edge of prolapse of at least $2 \mathrm{~cm}$ and an increase in symptoms were noted in the intervention group.

This study showed that POP remains relatively stable over time. Bothersome symptoms or functional impairment, not an anatomical progression of the prolapse, should drive the intervention.

Publisher's note Springer Nature remains neutral with regard to jurisdictional claims in published maps and institutional affiliations.

Alexandra Dubinskaya

a.dubinsky.md@gmail.com

Cedars-Sinai Medical Center, Los Angeles, CA, USA

2 St. Francis Medical Center, Hartford, CT, USA 